\title{
Um Modelo de Observatório para Projetos
}

\author{
Jeferson Kenedy Morais Vieira ${ }^{1,2}$ \\ Hermano Perrelli de Moura ${ }^{1}$ (orientador) \\ Ivaldir H. de Farias Júnior ${ }^{3}$ (coorientador) \\ ${ }^{1}$ Centro de Informática (CIn) - Universidade Federal de Pernambuco (UFPE) \\ Recife - PE - Brasil \\ ${ }^{2}$ Universidade Federal do Ceará (UFC) - Campus Quixadá \\ Quixadá - CE - Brasil \\ ${ }^{3}$ Universidade de Pernambuco (UPE) - Campus Garanhuns \\ Garanhunhs - PE - Brasil \\ jefersonkenedy@ufc.br, hermanodufpe.br, ivaldir.fariasdupe.br
}

\begin{abstract}
Resumo. $O$ ambiente de negócios atual é complexo, e a quantidade de informações disponíveis para tomada de decisões pode sobrecarregar os gerentes de projetos, e fazê-los perder de vista informações relevantes. Neste cenário, acredita-se que as organizações e os projetos podem se beneficiar com os produtos e serviços de informação oferecidos pelos observatórios. Assim, o problema que essa pesquisa busca solucionar é: de que forma, e a partir de quais elementos, podem ser estruturados observatórios para apoiar o compartilhamento de informações, a tomada de decisão, a gestão do conhecimento e a accountability em projetos? Para responder a essa questão de pesquisa, este trabalho tem como objetivo principal, a proposição de um modelo de observatório para projetos. O método de pesquisa principal escolhido para guiar a execução desta pesquisa é o Design Science Research (DSR). Além do DSR, mais duas metodologias de apoio serão utilizadas: Pesquisa-Ação e Mapeamento Sistemático da Literatura. As principais contribuições deste trabalho serão: o modelo de observatório para projetos e sua instância. Além disso, os resultados do mapeamento sistemático da literatura poderão ser considerados também como uma contribuição para a comunidade científica da área.
\end{abstract}

Keywords. Observatório; Modelo de Observatório; Projetos; Tomada de Decisão; Gestão da Informação e do Conhecimento; Compartilhamento de Informação.

Nível: Doutorado

Ano de ingresso no programa: 2018

Época prevista de conclusão: Fevereiro/2022

Data prevista da aprovação da proposta de tese: Fevereiro/2020

Eventos Relacionados: SBES 


\section{Introdução e Caracterização do Problema}

A arte de gerenciar projetos faz parte do cotidiano da maioria das pessoas. Seja no âmbito pessoal ou profissional, todos possuem projetos e/ou fazem parte direta ou indiretamente de algum. Os projetos são um dos componentes mais importantes das organizações de hoje. Em quase todas as empresas e setores, as organizações estão se tornando cada vez mais baseadas em projetos [Hazir 2015].

O ambiente de negócios atual é complexo, os gerentes de projeto precisam tomar decisões rápidas, alocar recursos escassos com eficiência e ter um foco claro. A quantidade de informações disponíveis para tomada de decisões pode sobrecarregar os gerentes de projetos, e fazê-los perder de vista informações relevantes [Caniëls and Bakens 2012, Marques et al. 2010]. Essa complexidade e a abundância de informações dificultam ainda a adoção de sistemas ou serviços de informação que permitam identificar cenários, ameaças e/ou oportunidades para as organizações. Torna-se complicado identificar e administrar dados e informações relevantes para seu negócio nesse caos informacional [Trzeciak 2009].

Não bastasse a grande quantidade de informações que precisam ser tratadas ao gerenciar um projeto, é comum que os gerentes atuem em mais de um projeto de forma simultânea, e em ambientes como esses, as informações disponíveis para o gerente são multiplicadas pelo número de projetos executados simultaneamente [Caniëls and Bakens 2012]. Essas dificuldades com o tratamento do excesso de informação geradas nos projetos também são sentidas a nível de programas e portfólios de projetos. De acordo com [Castro and Carvalho 2010], muitos modelos de gestão de projetos e portfólios utilizam muitas informações, que sobrecarregam os executivos das organizações que, consequentemente, não conseguem utilizá-las da forma adequada.

$\mathrm{Na}$ tentativa de facilitar o tratamento dessas informações, os gerentes buscam apoio nos sistemas de informações de gerenciamento de projetos (PMIS, do inglês, Project Management Information Systems). Para [Caniëls and Bakens 2012], esses sistemas devem fornecer aos gerentes de projeto suporte para a tomada de decisões para planejar, organizar e controlar projetos. No entanto, uma pesquisa feita por [Caniëls and Bakens 2012] demonstrou que a maioria dos gerentes está insatisfeita com as informações produzidas por esses sistemas. E uma das principais causas dessa insatisfação, de acordo com os autores, pode estar exatamente no excesso de informações que os gerentes precisam lidar durante os projetos.

Somando-se a dificuldade dos gestores em administrar essa sobrecarga de informações vivida durante o planejamento e a execução dos projetos, os gerentes e sua equipe precisam recuperar informações e conhecimento de projetos anteriores, buscando aprender com experiências vividas em outras situações semelhantes. Para [Batista et al. 2017b], o compartilhamento do conhecimento faz com que ocorra a inovação e aprendizagem contínua, promovendo um benefício mútuo. Nesse sentido, é importante que as organizações promovam a criação de uma cultura de compartilhamento para ampliar o conhecimento da organização. [Ajmal et al. 2010] afirmam que muitas empresas baseadas em projetos não possuem a expertise para lidar com seus ativos de conhecimento (especialmente aqueles obtidos com a experiência de projetos anteriores).

[Shinoda 2012] acrescenta que as características de temporariedade e singula- 
ridade dos projetos tornam o gerenciamento do conhecimento em organizações baseadas em projetos um desafio, pois é preciso garantir que os conhecimentos criados a cada projeto não se encerrem com término dos mesmos. Neste sentido, sistemas de gestão do conhecimento são fundamentais para organizações baseadas em projetos [Ajmal et al. 2010]. Dentre os sistemas de conhecimento contemporâneos encontram-se os observatórios [Batista et al. 2017a]. Para [Ortega and Valle 2010], estes observatórios respondem a uma crescente demanda por fontes integradas de informação, provendo dados acessíveis e confiáveis e facilitando o acesso à informação e ao conhecimento.

Não há uma definição de consenso na literatura para o conceito de "observatórios", embora quase todos os estudos afirmem que eles podem apoiar os processos de tomada de decisão estratégicos de uma organização, em diferentes áreas de geração de valor [Batista et al. 2017a, Batista et al. 2017b, Batista et al. 2016, Nascimento 2007, Ortega and Valle 2010, Soares et al. 2018]. De acordo com [Ortega and Valle 2010], os observatórios implementam sistemas de informação de dados abrangentes, confiáveis e acessíveis de diferentes fontes. A principal função dos observatórios é facilitar a transferência e o acesso à informação e ao conhecimento, a fim de: fomentar o debate, promover o diálogo, contribuir para a reflexão e estimular a criação de pensamento; facilitar a investigação; e melhorar o processo de tomada de decisão. Em um contexto público, os observatórios podem ser ainda excelentes ferramentas para promover a accountability [Schommer and Moraes 2010].

Diante do cenário apresentado, o problema que essa pesquisa busca solucionar é: de que forma, e a partir de quais elementos, podem ser estruturados observatórios para apoiar os projetos? Para responder a essa questão de pesquisa, este trabalho tem como objetivo principal, a proposição de um modelo de observatório para projetos. Perspectivando atingir esse objetivo primário, os seguintes objetivos específicos foram delineados: (i) identificar elementos que contribuam para o desenvolvimento de um modelo de observatório para projetos; (ii) definir as diretrizes que um observatório aplicado a projetos deverá seguir; e (iii) instanciar o modelo proposto com a construção de um observatório de projetos.

\section{Fundamentação Teórica}

Esta seção tem como objetivo apresentar as bases teóricas que fundamentam a construção desta pesquisa: observatórios e projetos.

\subsection{Observatórios}

O conceito de observatório, inicialmente associados à fenômenos naturais, foi transportado para a área social [Trzeciak 2009], em uma pesquisa rápida na internet é possível encontrar um vasto leque de observatório aplicados em contextos sociais. De acordo com [Trzeciak 2009], a área empresarial também passou a se beneficiar com o desenvolvimento dos observatórios, uma vez que eles são apresentados como ferramentas responsáveis pela coleta e difusão de informações estratégias para auxiliar nos processos de tomada de decisão. Como se pode ver, o termo sofreu uma expansão recente de seu significado ou de seu campo semântico desde sua atribuição original aos observatórios astronômicos [Beuttenmuller 2007].

Os observatórios passam a assumir um papel fundamental na sociedade do conhecimento, após se tornarem instrumentos de apoio ao estudo de fenômenos naturais. Para 
[Trzeciak 2009], no contexto da sociedade do conhecimento, a informação é um importante ativo, subsidiando e embasando o processo decisório, tornando-se fator crítico na gestão dos negócios. No entanto, as empresas encontram dificuldades para obter dados atualizados e confiáveis do seu ambiente competitivo, o que compromete consideravelmente a sua análise e o seu uso estratégico. Neste cenário, acredita-se que as empresas podem se beneficiar com os produtos e serviços de informação oferecidos pelos observatórios [Trzeciak 2009].

Dentre o variado "leque" de atividades que podem ser desenvolvidas e de possíveis produtos ofertados pelos observatórios, é possível enumerar: gestão e resultados em termos de conhecimento, informação e documentação; construção, monitoramento e armazenamento de sistemas de indicadores; condução e publicação de pesquisas e estudos especializados; manutenção e disponibilização de um banco ou base de dados; produção de notícias ou reprodução a partir de outras fontes; disponibilização de sistemas interativos para participação e comunicação online entre usuários e destes com o observatório. Deve-se registrar que a variedade de produtos gerados depende da finalidade do observatório e do público-alvo - nem todos os produtos listados acima são disponibilizados por um mesmo observatório [Silva et al. 2013].

\subsection{Projetos: relevância e desafios}

De acordo com o [PMI 2018], um projeto é um esforço temporário empreendido para criar um produto, serviço ou resultado exclusivo. Projetos movem o negócio para a inovação e a mudança; de fato, a única maneira de mudar uma organização, implementar uma estratégia, inovar, ou ganhar vantagem competitiva é através dos projetos [Shenhar and Dvir 2007].

No atual cenário, muitos são os desafios encontrados no contexto dos projetos e de seu gerenciamento, destacamos aqui a dificuldade do gestor de projetos em lidar com o excesso de informação disponível no ambiente de projetos, que dificulta o processo de tomada de decisão [Caniëls and Bakens 2012, Marques et al. 2010, Castro and Carvalho 2010]. Soma-se a isso, a dificuldade dos projetos em lidar com seus ativos de conhecimento, especialmente no que tange o compartilhamento de informações e conhecimento [Shinoda 2012, Todorovic et al. 2014, Ajmal et al. 2010].

No contexto de projetos públicos, a necessidade de transparência dos dados é vista como mais um desafio para os projetos. Os cidadãos precisam fiscalizar os projetos públicos não só em busca de corrupção, mas é preciso avaliar se o projeto foi concluído dentro do prazo e se atendeu ao orçamento estipulado, é preciso ainda que a população conheça quais os critérios que foram utilizados para priorizar este projeto em detrimento de outros [Valverde and Moore 2019].

\section{Comparação com Trabalhos Relacionados}

Foram identificados na literatura alguns trabalhos relacionados, os que mais se aproximaram dos objetivos desta pesquisa são apresentados aqui.

Em [Parreiras and Antunes 2012] encontramos um modelo de observatório de tendências para um centro de P\&D. [Schmidt and Silva 2018] também apresentam um modelo de observatório de tendências, mas para instituições de ciência e tecnologia. [Trzeciak 2009] propõe a criação de um modelo de observatório para arranjos produtivos 
locais. O trabalho de [Gomes et al. 2016] apresenta uma ontologia de domínio para observatórios com foco na indústria. Já em [Brown et al. 2016] é proposto uma taxonomia para observatórios web. [Sell et al. 2018] propõe a criação de um observatório para apoiar uma rede de expertise e inovação de um parque tecnológico. Já [Silva 2008] constrói um observatório de empresas de software no Brasil com recursos da web semântica.

Os trabalhos de [Trzeciak 2009], [Parreiras and Antunes 2012] e [Schmidt and Silva 2018] apresentam modelos para observatórios, já [Gomes et al. 2016] e [Brown et al. 2016], propõem, respectivamente, a criação de uma taxonomia e de uma ontologia para observatórios, no entanto, nenhum deles propõe a construção de um modelo de observatórios com foco em projetos, que é o objetivo desta pesquisa. Finalmente, os trabalhos de [Sell et al. 2018] e [Silva 2008] apresentam instâncias de observatórios, o trabalho aqui apresentado diferencia-se destes, pois propõe além da construção de uma instância de observatório aplicado a projetos, a criação de um modelo de observatório para projetos.

\section{Metodologia}

O objetivo desta seção é apresentar os métodos que serão utilizados na condução desta pesquisa. Para isso, são apresentados: uma categorização e as etapas da pesquisa.

\subsection{Caracterização da Pesquisa}

Foi utilizada a taxonomia de [Wohlin and Aurum 2015], para classificar os resultados de pesquisas intermediárias ou a pesquisa como uma unidade inteira. Considerando os resultados da pesquisa, este trabalho é considerado uma pesquisa aplicada. No que diz respeito a natureza lógica, essa pode ser considerada uma pesquisa do tipo indutiva. Com relação ao objetivo da pesquisa, este trabalho pode ser considerado tanto uma pesquisa exploratória, como uma pesquisa descritiva. Considerando a abordagem de pesquisa, utiliza-se a filosofia pragmática para a condução desta pesquisa.

O método de pesquisa principal escolhido para guiar a execução desta pesquisa é o Design Science Research (DSR). Além do DSR, mais duas metodologias de apoio serão utilizadas: Pesquisa-Ação e Mapeamento Sistemático da Literatura. No que diz respeito ao processo de pesquisa, este trabalho pode ser considerado uma pesquisa qualitativa. Como métodos de coleta de dados esta pesquisa utilizará: entrevistas, pesquisa documental e bibliográfica, observação do participante e survey. E, finalmente, esta pesquisa utilizará a análise temática como método de análise dos dados.

\subsection{Etapas da Pesquisa}

[Hevner 2007] apresenta um framework de pesquisa em sistemas de informação utilizando DSR com o foco em três ciclos de pesquisa: relevância, rigor e design. Ainda de acordo com [Hevner 2007], esses três ciclos devem estar presentes e claramente identificáveis em um trabalho que utilize o DSR como método de pesquisa. Os Ciclos de Relevância devem preencher o ambiente contextual do projeto de pesquisa com as atividades da ciência do design. Os Ciclos do Rigor devem conectar as atividades da ciência do design com a base de conhecimento de fundamentos científicos, experiências e expertises. Os Ciclos de Design devem iterar entre as principais atividades de construção e avaliação dos artefatos e processos de design da pesquisa [Hevner 2007]. A figura 1 
apresenta as onze etapas que deverão ser executadas para a conclusão desta pesquisa, e identifica os ciclos de DSR apontados por [Hevner 2007].

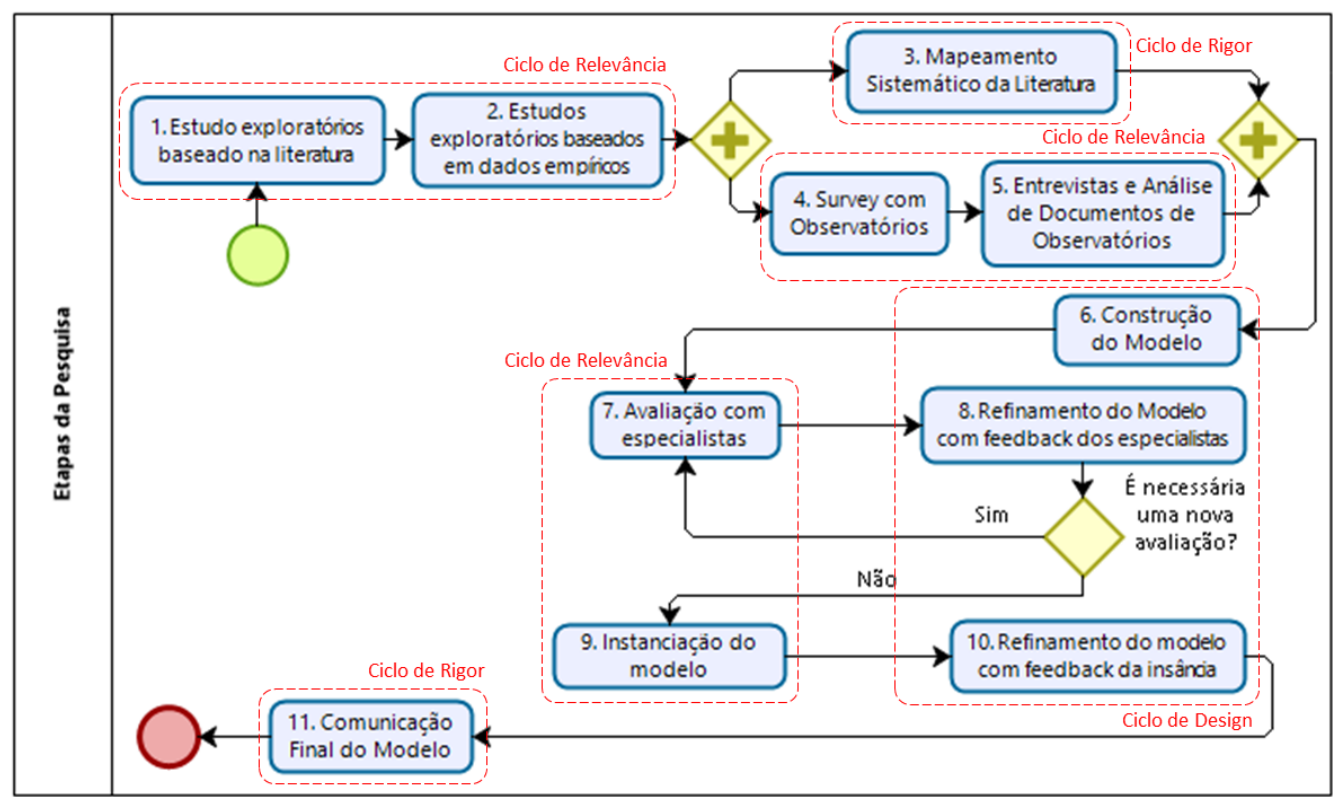

Figura 1. Etapas da pesquisa. Fonte: Próprio autor.

A primeira e a segunda etapa fazem parte do ciclo de relevância do DSR. A primeira etapa teve como objetivo a realização de um estudo exploratório ad hoc da literatura com o objetivo de compreender como os observatórios poderiam contribuir para os projetos. A segunda etapa tem como objetivo a realização de alguns estudos empíricos de cunho exploratório, com o objetivo de avaliar a aplicação de observatórios em projetos.

A terceira etapa da pesquisa corresponde ao ciclo de rigor do DSR. Esta etapa consiste na execução de um mapeamento sistemático da literatura para coletar informações sobre observatórios e sua aplicação em projetos. A quarta e a quinta etapa, correspondem a mais um ciclo de relevância do DSR. Na quarta etapa será realizado um survey com observatórios com o objetivo de compreender seu funcionamento, bem como coletar dados para a construção do modelo aqui proposto. A quinta etapa também tem como objetivo a coleta de dados, no entanto, com maior profundidade que na etapa anterior, para isso serão realizadas entrevistas e análises de documentos de observatórios existentes. Tanto o survey como as entrevistas serão realizados com gestores e/ou representantes de observatórios existentes.

As etapas 6, 8 e 10 fazem parte do ciclo de design do DSR. Já as etapas 7 e 9 fazem parte de mais um ciclo de relevância do DSR. A partir dos dados coletados, uma primeira versão do modelo de observatório para projetos será construído na sexta etapa. Em seguida, na sétima etapa, este modelo passará por uma avaliação com especialistas, através de grupos focais. Então, o modelo passará, na oitava etapa, por um refinamento a partir dos feedbacks coletados na etapa 7. Caso necessário, uma nova avaliação por especialistas será realizada até que o modelo construído esteja maduro o suficiente para ser instanciado. Na nona etapa, o modelo deverá ser instanciado através de uma pesquisaação, e um observatório de projetos deverá ser criado a partir do modelo proposto. A etapa 11 corresponde a mais um ciclo de rigor do DSR, e tem como objetivo a comunicação 
final do modelo. Neste momento, essa pesquisa encontra-se na etapa 2 da Figura 1. O protocolo da etapa 3 (mapeamento sistemático da literatura) já foi construído e avaliado.

As etapas 1, 2, 3, 4, e 5 ajudarão a identificar os elementos que podem contribuir para o desenvolvimento de um modelo de observatório para projetos, atendendo ao primeiro objetivo específico desta pesquisa. Nas etapas $6,7,8,9,10$ serão definidas, refinadas e avaliadas as diretrizes que um observatório aplicado a projetos deverá seguir, que corresponde ao segundo objetivo específico deste trabalho. E a etapa 9 atende ainda ao terceiro objetivo específico deste trabalho, instanciando o modelo proposto.

\section{Resultados Preliminares}

O estudo exploratório da literatura (primeira etapa da Figura 1) contribuiu para identificarmos uma lacuna na literatura sobre a aplicação de observatórios em projetos. Em seguida, na etapa 2, foi proposta a execução de alguns estudos empíricos de cunho exploratório, até o momento dois estudos já foram realizados e um está em execução. O primeiro estudo foi desenvolvido em uma disciplina do Programa de Pós-Graduação em Ciência da Computação do Centro de Informática (CIn) da Universidade Federal de Pernambuco (UFPE), onde foi solicitado que os alunos concebessem um Observatório Universal de Projetos (OUP), como resultado desse estudo, foi construído um documento inicial de referência para o OUP, que pode ser encontrado em [Moura et al. 2018].

O segundo estudo também foi realizado no Centro de Informática da UFPE, mas desta vez com alunos do Mestrado Profissional (MPROF). Estes alunos eram servidores dos Institutos Federais de Educação, Ciência e Tecnologia (IF) e da Universidade Federal de Pernambuco (UFPE). Foi solicitado aos alunos a construção de cinco observatórios de projetos para os IFs (um para cada região do Brasil) e um para a UFPE. Foram coletados dados de 1.078 projetos, e esta experiência trouxe algumas reflexões sobre o ambiente de projetos dessas instituições: (i) não existe uma base centralizada com informações sobre os projetos das instituições; (ii) muitos projetos não são gerenciados e nem tratados como projetos; e (iii) em boa parte das instituições não há um processo formal de gerenciamento de projetos. Essa experiência também proporcionou algumas reflexões sobre a construção de observatórios de projetos: (i) ferramentas de coleta automatizada de dados podem facilitar a construção de observatórios; (ii) os dados sobre os projetos devem ser coletados continuamente, para evitar que os observatórios representem apenas uma imagem estática dos projetos em um determinado momento do tempo; (iii) análise simples podem ser feitas utilizando ferramentas simples (como planilhas, formulários, entre outros), no entanto para evitar que os observatórios sejam apenas repositórios de dados é necessário utilizar ferramentas mais robustas de extração de conhecimento em bases de dados; e (iv) houve resistência para o compartilhamento dos dados sobre os projetos por parte de alguns gestores, deve-se criar estratégias para que isso não inviabilize a construção do observatório.

\section{Contribuições}

Para [Hevner et al. 2004], a contribuição de uma pesquisa que utiliza como método o DSR é, muitas vezes, o próprio artefato construído, e esses artefatos devem ser implementáveis, daí a importância de instanciar artefatos na ciência do design. Neste sentido, as principais contribuições deste trabalho são: o modelo de observatório para projetos e sua instância. Além disso, os resultados do mapeamento sistemático da literatura podem ser considerados também como uma contribuição para a comunidade científica da área. 


\section{Referências}

Ajmal, M., Helo, P., and Kekäle, T. (2010). Critical factors for knowledge management in project business. Journal of Knowledge Management, 14(1):156-168.

Batista, A. D., Pacheco, R. C. S., Duarte, K. B., Sell, D., and Marchezan, M. A. (2016). Observatórios de Competências. In VI Congresso Internacional de Conhecimento e Inovação (ciKi), Bogotá.

Batista, A. D., Pacheco, R. C. S., Duarte, K. B., Sell, D., and Marchezan, M. A. (2017a). Observatórios de Conhecimento: conceitualização e perspectivas de aplicação na Gestão do Conhecimento. Produção em Foco, 7(1):50-63.

Batista, A. D., Schneider, V., Sell, D., and Pacheco, R. C. S. (2017b). Processo de Engenharia do Conhecimento para Observatórios. In VII Congresso Internacional de Conhecimento e Inovação, Foz do Iguaçu.

Beuttenmuller, G. (2007). Observatório Locais de Políticas Públicas no Brasil: seu papel na produção, disseminação e transparência das informações. (Dissertação de Mestrado - Fundação Getúlio Vargas).

Brown, I. C., Hall, W., and Harris, L. (2016). Towards a taxonomy for web observatories. In Proceedings of the 23rd International Conference on World Wide Web, pages 10671072, Seoul, Korea. ACM.

Caniëls, M. C. and Bakens, R. J. (2012). The effects of Project Management Information Systems on decision making in a multi project environment. International Journal of Project Management, 30(2):162-175.

Castro, H. G. D. and Carvalho, M. M. D. (2010). Gerenciamento do Portfolio de Projetos: um estudo exploratório. Gestão Produção, 17(2):1-15.

Gomes, M. S., Rossari, T., Ecker, G., Visintin, L., and Candido, A. P. (2016). Uma ontologia de domínio no contexto de observatórios. In IX ONTOBRAS Seminário de Pesquisa em Ontologias do Brasil, volume 1862, pages 203-208, Curitiba, PR.

Hazir, O. (2015). A review of analytical models, approaches and decision support tools in project monitoring and control. International Journal of Project Management, 33(4):808-815.

Hevner, A. R. (2007). A Three Cycle View of Design Science Research. Scandinavian Journal of Information Systems, 19(2).

Hevner, A. R., March, S. T., Park, J., and Ram, S. (2004). Design Science in Information Systems Research. MIS quarterly, 28(1):77-2004.

Marques, G., Gourc, D., and Lauras, M. (2010). Multi-criteria performance analysis for decision making in project management. International Journal of Project Management, 29(8):1057-1069.

Moura, H. P. d., Luna, A. J. H. d. O., and Farias Junior, I. H. d. (2018). Concepção do Observatório Universal de Projetos: Documento de Referência Inicial. Technical report, Universidade Federal de Pernambuco, Recife.

Nascimento, M. E. M. (2007). Mapeamento e Análise de Instituições Congêneres. Technical report, CGEE - Centro de Gestão e Estudos Estratégicos, Brasília. 
Ortega, C. and Valle, R. S. S. d. (2010). Nuevos retos de los observatorios culturales. Boletín GC: Gestión Cultural, (19):1-15.

Parreiras, V. M. A. and Antunes, A. M. d. S. (2012). Aplicação de Foresight e Inteligência Competitiva em um Centro de P\&D Empresarial por meio de um Observatório de Tendências: desafios e benefícios. Revista Gestão \& Conexões, 1(1).

PMI (2018). Guide to the Project Management Body of Knowledge (PMBOK Guide). Project Management Institute (PMI), sixth edit edition.

Schmidt, N. S. and Silva, C. L. (2018). Observatório como instrumento de prospectiva estratégica para as Instituições de Ciência e Tecnologia (ICTs). Interações (Campo Grande), 19(2):153.

Schommer, P. C. and Moraes, R. L. (2010). Observatórios sociais como promotores de controle social e accountability: reflexões a partir da experiência do Observatório Social de Itajaí. Gestão.Org - Revista Eletrônica de Gestão Organizacional, 8(3):298326.

Sell, D., Batista, A. D., and Todesco, J. L. (2018). Knowledge observatories: a case study. Revista Democracia Digital e Governo Eletrônico, 1(17):82-91.

Shenhar, A. J. and Dvir, D. (2007). Reinventing Project Management: the diamond approach to successful growth and innovation. Harvard Business School Press, Boston.

Shinoda, A. C. M. (2012). Gestão do conhecimento em projetos: um estudo sobre conhecimentos relevantes, fatores influenciadores e práticas em organizações projetizadas (Dissertação de Mestrado - Universidade de São Paulo).

Silva, A. L. d., Netto, M., Helou Filho, E. A., and Selig, P. M. (2013). Observatórios de informação e conhecimento: discutindo bases conceituais e perspectivas de efetividade. In IX Congreso Nacional de Excelencia em gestão.

Silva, M. M. (2008). Desenvolvimento de um Observatório de Empresas de Software no Brasil com Recursos da Web Semântica. (Dissertação de Mestrado - Universidade FUMEC).

Soares, L. C., Ferneda, E., and Prado, H. A. d. (2018). Observatórios: um levantamento do estado do conhecimento. Brazilian Journal of Information Science, 12(3):86-110.

Todorovic, M. L., Petrovic, D. C., Mihic, M. M., Obradovic, V. L., and Bushuyev, S. D. (2014). Project success analysis framework: A knowledge-based approach in project management. International Journal of Project Management, 33(4):772-783.

Trzeciak, D. (2009). Modelo de observatório para arranjos produtivos locais (Tese de Doutorado - Universidade Federal de Santa Catarina).

Valverde, M. and Moore, A. (2019). The performance of transparency in public-private infrastructure project governance: The politics of documentary practices. Urban Studies, 56(4):689-704.

Wohlin, C. and Aurum, A. (2015). Towards a decision-making structure for selecting a research design in empirical software engineering. Empirical Software Engineering, 20(6):1427-1455. 